\title{
Diseño SCADA para un prototipo seleccionador de piezas
}

\section{RESUMEN}

El prototipo seleccionador realiza una clasificación y distribución de piezas en función al color y tipo de material que tengan las piezas. La clasificación se realiza por dos sensores que, dependiendo del tipo de pieza a evaluar, pueden o no activarse. Un controlador lógico programable (PLC) tiene la función de controlar de manera automática todo el proceso a través de un programa. Este programa permite que la distribución de las piezas se realice hacia distintas rampas en donde serán almacenadas. El proceso puede ser iniciado fisicamente presionando un pulsador en el prototipo, como también al presionar un botón en la pantalla de una computadora. En la pantalla no solo se muestran botones, sino también se puede apreciar en tiempo real lo que ocurre durante el proceso en el prototipo seleccionador de piezas.

Palabras clave: Prototipo, seleccionador, SCADA.

SCADA DESING FOR A PROTOTYPE COUCH PARTS

\section{ABSTRACT}

The prototype performs a classification and distribution of parts according to color and type of material of the pieces. The classification is done by two sensors that depending on the object may or may not be activated depending of the type of piece. A programmable logic controller (PLC) has the function of automatically controlling the entire process through a program. This program allows the distribution of the parts to different ramps where they will be stored. The physical process may be initiated by pressing a button on the prototype, as well as through a button on a computer screen. The display shows not only buttons, you can see in real time what happens in the prototype parts selection.

Key words: Prototype, selector, SCADA

\section{INTRODUCCIÓN}

El presente artículo muestra el desarrollo del diseño para un prototipo seleccionador de piezas en un software SCADA. Ello se realizará con el software Intouch v10.1.

Con el prototipo implementado, se debe realizar la programación del PLC con el propósito de controlar de manera automática el proceso y, además, para que tenga la posibilidad de recibir señales que provengan de un SCADA. Esto es, para que no solamente sea controlado por una persona que está al costado del prototipo, sino estando a distancia, pudiendo ser controlado y monitoreado desde una computadora.

Este prototipo fue desarrollado por los alumnos María Julia Carpena Del Pozo y Lindembert Hernán Solís, para el curso "Procesos de Manufactura" asistido por computadora II, correspondiente al décimo ciclo de la carrera de Ingeniería Industrial de la Universidad Ricardo Palma. (Ver Fig 1: Prototipo seleccionador de piezas). 
COMPONENTES DEL PROTOTIPO

\begin{tabular}{|l|c|c|}
\hline Descripción & Cantidad & Especificación \\
\hline Plancha metálica & 1 & $900 \times 610 \mathrm{~mm}$ \\
\hline Ángulo & 9 & $285 \times 45 \mathrm{~mm}$ \\
\hline Rampa de almacenaje final & 3 & $3355 \times 60 \times 40 \mathrm{~mm}$ \\
\hline Almacén temporal de piezas & 1 & $210 \times 45 \times 45 \mathrm{~mm}$ \\
\hline Cilindro doble efecto 5/2 & 1 & $\varnothing: 12 \mathrm{~mm}$ carrera $50 \mathrm{~mm}$ \\
\hline Cubo & 6 & $25 \times 25 \mathrm{~mm}$ \\
\hline Faja transportadora & 1 & $1250 \times 50 \mathrm{~mm}$ \\
\hline Final de carrera & 1 & normalmente abierto \\
\hline Varilla fija & 2 & $140 \times 20 \mathrm{~mm}$ \\
\hline Cilindro simple efecto & 2 & $\varnothing: 20$ carrera $50 \mathrm{~mm}$ \\
\hline Sensor inductivo & 1 & montaje enrasado \\
\hline Sensor óptico & 1 & reflectivo \\
\hline Botonera & 1 & 5 botones, 1 Selector \\
\hline PLC FESTO & 1 & 16 inputs, 16 outputs \\
\hline Electro válvulas 5/2 & 1 & monoestable \\
\hline Electro válvula 3/2 & 2 & monoestable \\
\hline Estranguladora de caudal & 1 & $4 \mathrm{~mm}$ \\
\hline Manguera & $8 \mathrm{~m}$ & $\varnothing: 4 \mathrm{~mm}$ \\
\hline Manguera & $2 \mathrm{~m}$ & $\varnothing: 6 \mathrm{~mm}$ \\
\hline Motor & 1 & $24 \mathrm{~V}$ DC \\
\hline Faja & 104 & $370 \times 50 \mathrm{~mm}$ \\
\hline Pernos con tuerca & & $1 / 8 \times 1 " 32$ \\
\hline Tornillo autorroscante & 14 & \\
\hline
\end{tabular}

CARACTERÍSTICAS TÉCNICAS DEL PROTOTIPO

LAS CARACTERÍSTICAS TÉCNICAS DEL PROTOTIPO SON LAS SIGUIENTES:

\begin{tabular}{|l|r|}
\hline Capacidad del almacén temporal & 6 piezas \\
\hline Distancia máx. de conmutación sensor óptico & $500 \mathrm{~mm}$ \\
\hline Distancia máx. de conmutación sensor inductivo & $3 \mathrm{~mm}$ \\
\hline Velocidad de la faja & $5 \mathrm{~cm} / \mathrm{seg}$ \\
\hline Capacidad de rampas de almacenaje & 12 piezas \\
\hline Dimensiones total & $900 \times 610 \times 210 \mathrm{~mm}$ \\
\hline
\end{tabular}


Figura 1: Prototipo seleccionador de piezas.

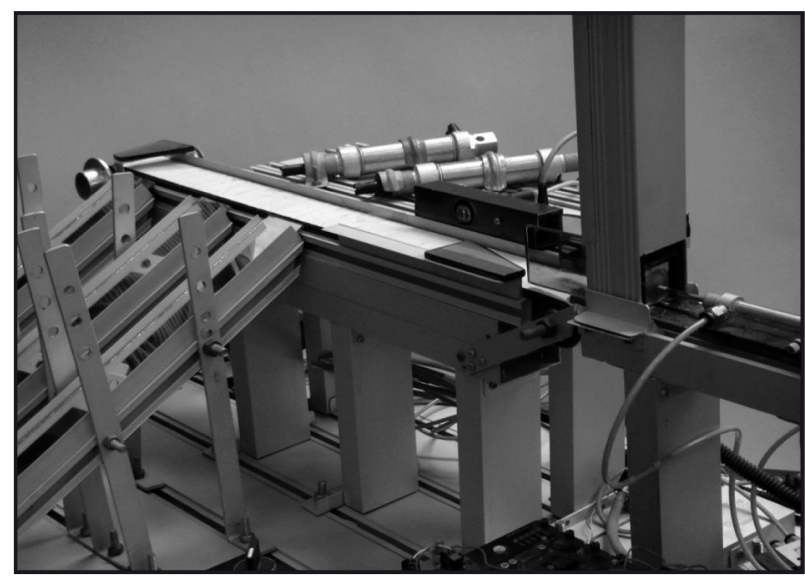

\section{DESCRIPCIÓN DE LAS ETAPAS DEL PROCESO}

\section{Almacén y alimentador de piezas}

El almacén vertical se usa para alojar las piezas que serán clasificadas según su color y material. Las piezas son apiladas manualmente una sobre otra sin importar su orden. En el interior del almacén, se ubica un final de carrera que indica la presencia de piezas. En caso el almacén no cuente con piezas, la siguiente etapa del proceso no se inicia y una señal de alerta se activa. (Ver Fig. 2: Almacén y alimentador de piezas).

Si existen piezas en el almacén, estas son expulsadas hacia una faja transportadora por medio de un cilindro de doble efecto. Para que el cilindro no dañe las piezas, su avance está regulado por una válvula estranguladora de caudal, de modo que su velocidad de expulsión de piezas se reduce. El cilindro es controlado por una electroválvula $5 / 2$ monoestable, la que permite su avance o retroceso según el programa de control.

Figura 2: Almacén y alimentador de piezas.

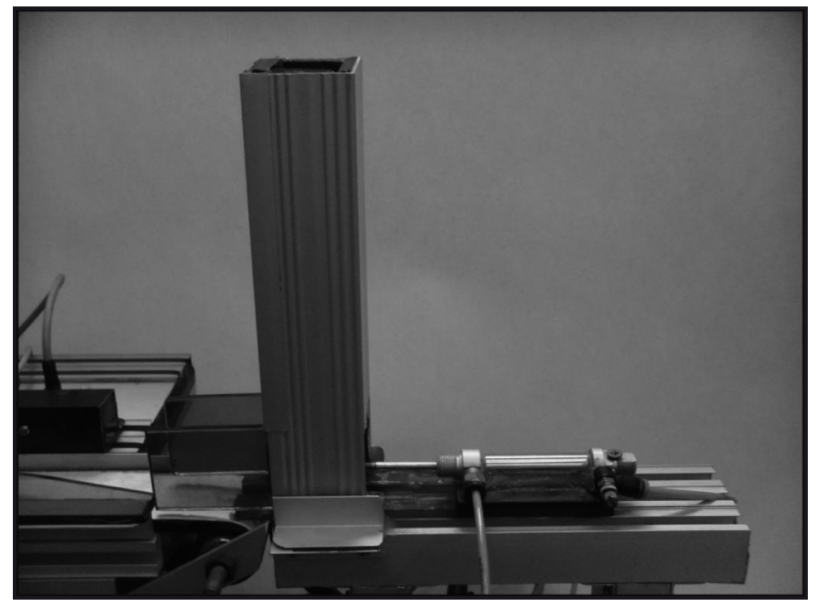

\section{Clasificación de piezas}

El clasificador consta de una faja transportadora accionada por un motor, un sensor inductivo de montaje enrasado y un sensor óptico de reflexión directa. Los sensores que se emplean son de proximidad sin contacto. Su principal característica es que convierten una magnitud física en una señal binaria con los estados "ON" o "OFF" (activado: 1 o desactivado: 0).

El sensor inductivo solo puede detectar materiales conductores de electricidad. Este tipo de sensores detectan piezas hasta de $250 \mathrm{~mm}$ de distancia, esto dependerá del diámetro del sensor y del material a evaluar (acero dulce, níquel cromo, latón, aluminio, etc.). El sensor óptico emplea medios ópticos y electrónicos para la detección de objetos, para ello utiliza luz roja o infrarroja. La distancia de detección del sensor que se empleará depende mucho de la reflectividad del objeto.

Las piezas que son expulsadas hacia la faja transportadora por el alimentador de piezas, pasan primero por el sensor inductivo y luego por el sensor óptico. Las piezas metálicas son detectadas por el sensor inductivo y por el sensor óptico. Las piezas acrílicas rojas son detectadas solo por el sensor óptico. Las piezas acrílicas de color negro mate no son detectadas por los sensores. (Ver Fig. 3: Sensores óptico e inductivo). En la siguiente tabla, se muestra la activación de los sensores en función al material y color de la pieza:

\begin{tabular}{|l|c|c|c|}
\hline & Rojo & Aluminio & Negro mate \\
\hline Sensor Inductivo & 0 & 1 & 0 \\
\hline Sensor Óptico & 1 & 1 & 0 \\
\hline
\end{tabular}

Figura 3: Sensores óptico e inductivo.

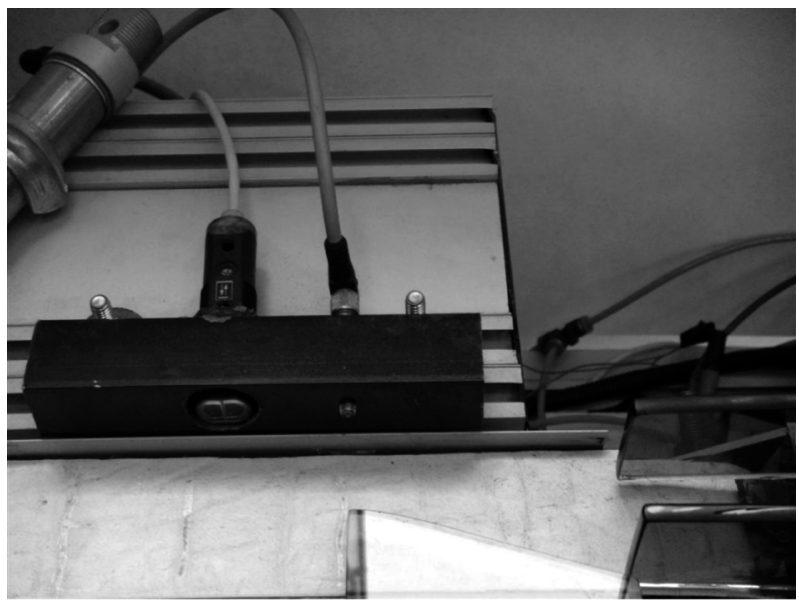




\section{Distribución de piezas}

Las piezas se clasifican en función a los sensores que se activan, por lo que de debe realizar un programa en un controlador lógico programable (PLC) que permite controlar los cilindros para que puedan extenderse y distribuir las piezas hacia las rampas de almacenaje, según el tipo de objeto. Una vez que las piezas hallan pasado a la rampa de almacenaje, el cilindro nuevamente retorna a su posición inicial.

En caso que la pieza sea de metal, se extiende el primer cilindro, si es roja se extiende el segundo y si es negro mate, ningún cilindro se extiende y la pieza llega al final de la faja y cae por una de las rampas. Ambos cilindros están conectados a electroválvulas $3 / 2$ monoestable a través de tubos flexibles. (Ver Fig. 4: Cilindros distribuidores de piezas).

\section{Rampas de almacenaje}

Existen tres rampas por las que caen y se almacenan las piezas. La primera rampa almacena las piezas de aluminio, la segunda, las de color rojo y, la última, las de color negro mate. Cada una de estos almacenes alberga 12 piezas. (Ver Fig 5: Rampas de almacenaje de piezas)

\section{Programación del PLC (Statement List)}

La programación del PLC, se ha realizado en el lenguaje lista de instrucciones. Se ha empleado un programa principal y dos subprogramas (Inicio y reseteo). La parada del proceso se ha realizado dentro del programa principal. (Ver Fig 6: Ventanas del software de programación del PLC). Se dispone de una botonera con tres pulsadores (Start, Stop y Reset). Según la programación del PLC, existen 2 formas de iniciar la secuencia del proceso: pre-

Figura 4: Cilindros distribuidores de piezas.

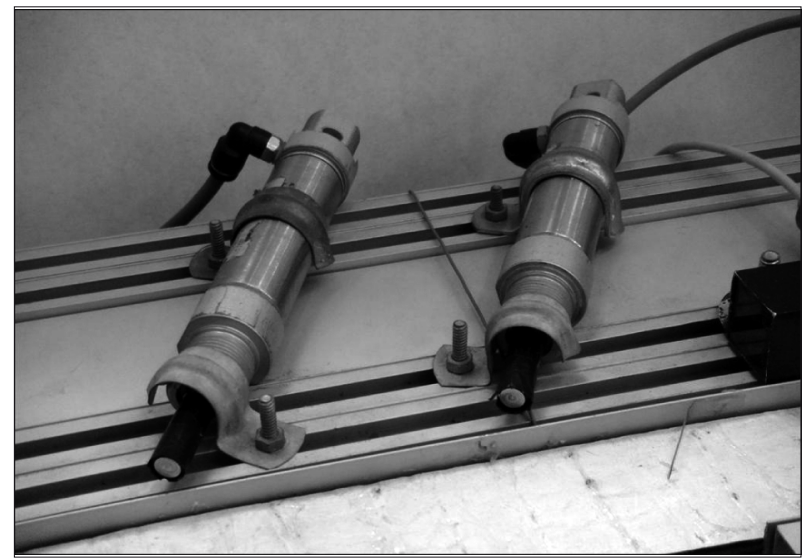

Figura 5: Rampas de almacenaje de piezas.

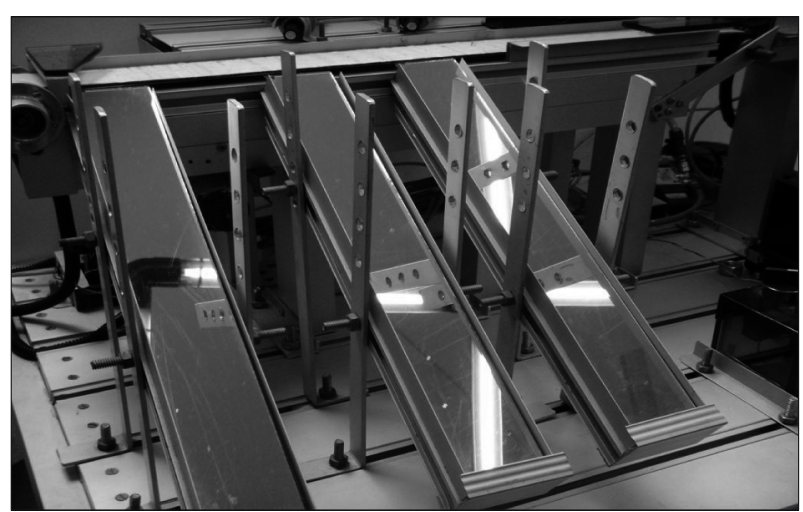

sionando "Start" o presionando el botón verde en la pantalla del SCADA llamado "Start", el cual está configurado para que active la señal M1.0 (marca_ start). Para detener la secuencia, se presiona el botón "Stop" o desde la pantalla el botón rojo "Stop". Este botón activa la señal M1.1 (marca_stop). El reseteo del proceso se realiza con el botón "Reset" o desde la pantalla con el botón amarillo del mismo nombre (M1.4).

\section{Diseño SCADA para el prototipo}

SCADA es una aplicación de software especialmente diseñada para funcionar sobre computadoras en el control de producción, proporcionando comunicación con los dispositivos de campo (sensores y actuadores) y controlando el proceso de forma automática desde la pantalla de la computadora. Puede proveer información que se genera en el proceso productivo a diversos usuarios dentro de la empresa (supervisión, control calidad, control de producción, almacenamiento de datos, etc.).

El diseño de la pantalla de supervisión y control del usuario se ha desarrollado con el software InTouch

Figura 6: Ventanas del software de programación del PLC

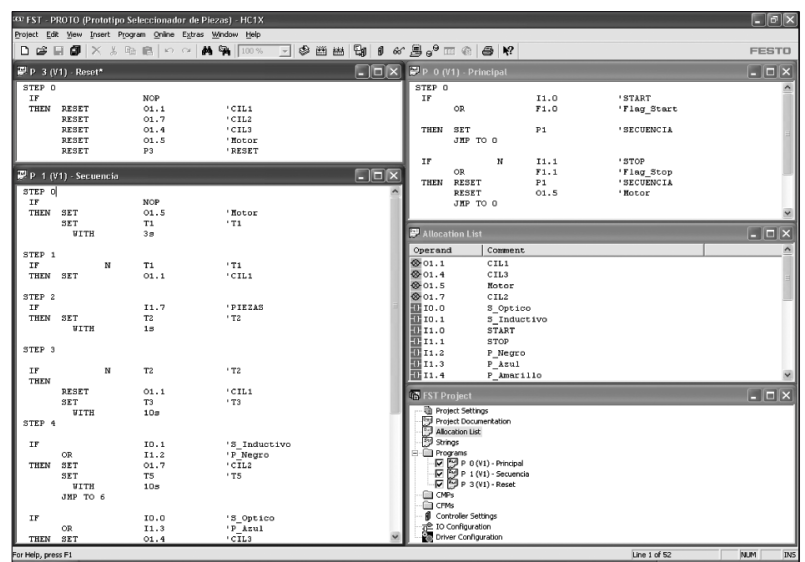


v10.1 de la empresa Wonderware, el cual permite enlazarnos con el PLC y, desde sus ventanas, interactuar con su programación. Para ello, es indispensable utilizar el programa de comunicación IPC Data Server, el cual nos permite realizar la comunicación DDE entre la computadora y el PLC. El IPC Data Server envía y recibe datos de los PLC's que tengan instalado el driver TCP/IP (IPC's) así como con PLC's que tengan solo conexión RS232 (FPC's). También actúa como un servidor DDE (Dinamic Data Exchange), que provee una interfase con los programas clientes DDE, en este caso, el InTouch.

EI IPC Data Server se puede comunicar con 48 PLC's utilizando TCP/IP. Estos deben tener el driver TCP/IP instalado y funcionando. Además, el IPC Data Server puede comunicarse vía RS232 con 4 PLC's.

El diseño de la aplicación SCADA tiene tres ventanas. Una es la principal, que muestra todo el prototipo en una vista en 3D, y las otras ventanas corresponden a vista frontal y lateral del prototipo. (ver Figura 7).

\section{CONFIGURACIÓN DEL SCADA}

Se crearon variables en la ventana del Tagname Dictionary que permitan el control y supervisión del proceso. Las variables se configuran tipo I/O Discrete y la ruta de acceso (Access Name) entre PLC y SCADA se hará a través del IPC_DATA (ver Figura 8).

Una vez concluida la configuración, se ingresa a modo ejecución "runtime" y se logra monitorear el

Figura 7. Diseño del prototipo seleccionador de piezas

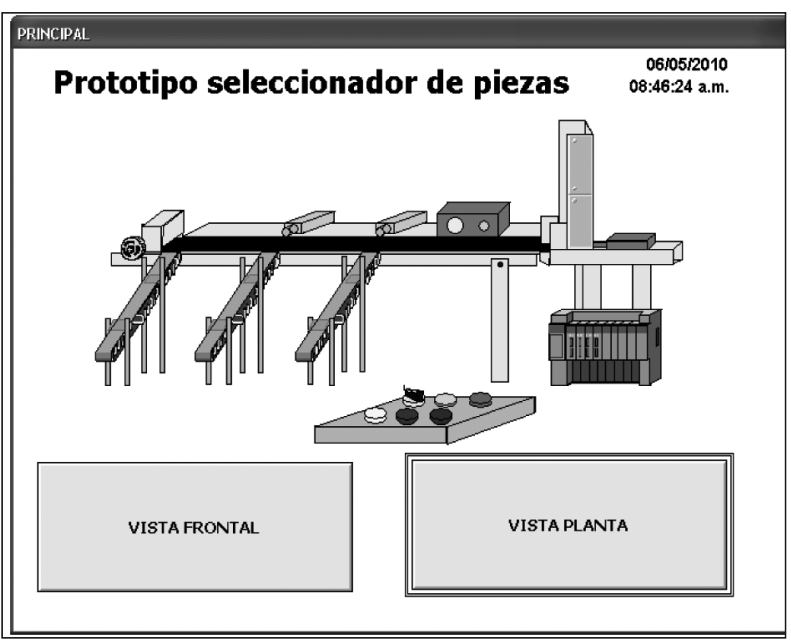

Figura 8: Ventana Tagname Dictionary

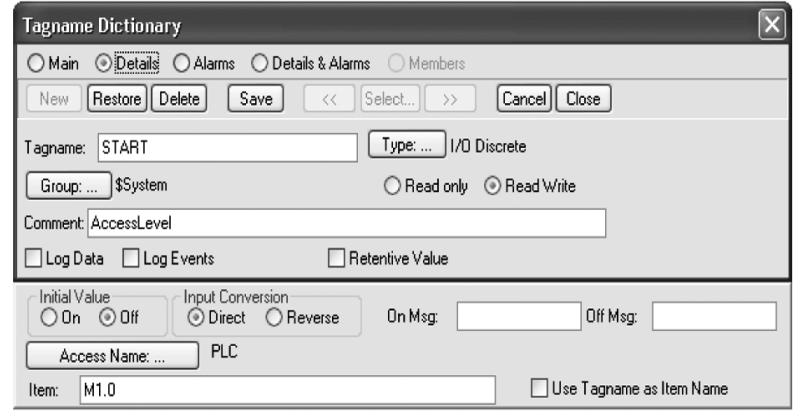

proceso; además, se puede controlar su secuencia desde los botones que se muestran en la pantalla principal.

\section{CONCLUSIONES}

1. Antes de diseñar y configurar una aplicación SCADA para cualquier proceso, este debe funcionar correctamente y controlado por un PLC.

2. La programación del PLC puede variar cuando se desea comunicarlo con un software SCADA, esto debido a que las señales de inicio, parada y reset se activaran a través de la computadora y no de señales físicas.

3. El diseño de la aplicación SCADA debe ser semejante al proceso real.

4. La configuración que se realiza en el SCADA es para definir las variables que intervienen en el proceso y la inteface de comunicación entre PLC y SCADA.

5. El software Intouch permite una correcta comunicación con el prototipo, permitiendo el monitoreo en tiempo real de lo que ocurre en el proceso.

\section{REFERENCIAS BIBLIOGRÁFICAS}

1. Rodriguez, Aquilino. (2006). Sistemas SCADA. Madrid, Marcombo.

2. Cembranos F. J. (2002). Sistemas de control secuencial. Madrid, Paraninfo.

3. Deppert W. y Stoll K. (2001). Dispositivos neumáticos. México D.F., Alfaomega.

4. FST User's Guide. Festo.

5. InTouch Users Guide. Invensys Systems. Wonderware.

6. Velásquez J. (2005). Los sensores en la producción. Revista de la Facultad de Ingeniería de la Universidad Ricardo Palma; pp. 112-116. 